$$
\text { بداية العهدالجديدفي تاريخ النحو العر بي بالإمام الفراهي }
$$

\title{
BEGINNING OF NEW ERA IN THE HISTORY OF ARABIC GRAMMAR WITH IMAM AL-FARAHI
}

$$
\text { * الدكتور أورنك زيب الأعظمي }
$$

DOI: 10.29370/siarj/issue3ar8

Link: https://doi.org/10.29370/siarj/issue3ar8

\begin{abstract}
:
India has been contributing a lot to the Arabic language and literature since its arrival to the sub-continent. Its scholars, both Hindus and Muslims, did their best to enrich Arabic and to promote it on their motherland. Even they used the Arabic language as their secret language. Numerous scholars from different states and districts emerged to develop Arabic, its sciences and arts. They wrote books, both original and translation, and opened madrasah, colleges and universities to teach Arabic language and literature. From Azamgarh, which is a small district of India and a famous centre of Arabic and Islamic Studies, emerged several scholars to serve Arabic and to contribute to its development. Among such scholars there is one scholar, 'Allamah Abd-ul-Hamid alFarahi, whose name and fame crossed the boundaries of the nation. He became famous in the Arab world for his valuable contributions towards knowledge and different sciences. As regards Arabic he wrote books in Arabic, composed poems in Arabic and developed Arabic grammar. His treatises and pamphlets are so famous in this regard. They are taught in the India madrasas and are also prescribed in the Indian colleges and universities. He invented some new things and introduced some new methods in this field which are presented in this short article.
\end{abstract}

KEYWORDS: Al-Farahi Arabic, language, Grammar, literature, contributions

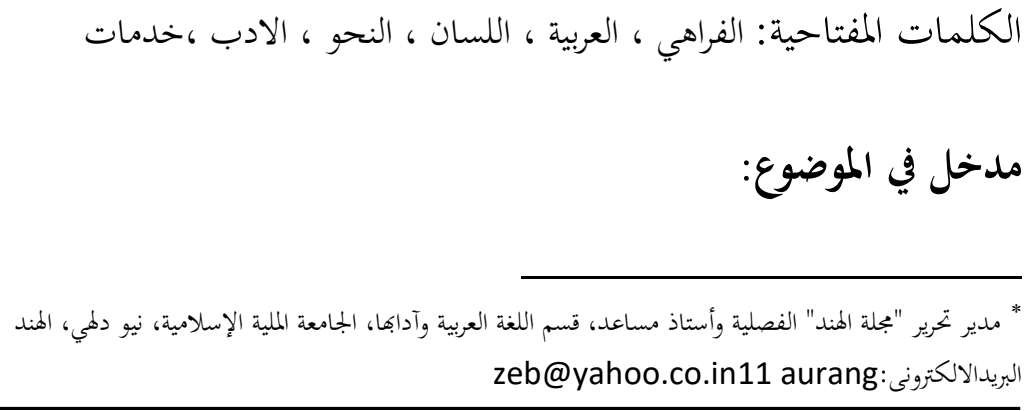


قد أبرز الله تعالى في كل عهد شخصيات كبرى أحيت العلوم ونفخت الروح فيها وتركت مآثر قيّمة في

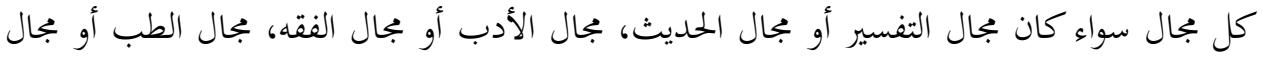
الحساب. ومن هذه المجالات المختلفة مجال النحو العربي وهو بجال مهمّ بحيث كونه سلّمًا إلى العلوم

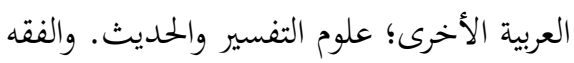

وفي هذا المجال برز عدد كبير من الرجال الذين ذاع صيتهم وأولمم سيبويه وهو الذي صنّف كتابًا منفردًا

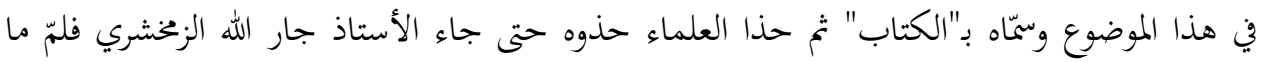
شتّوه وجمع ما فرّقوه وصنّف كتابًا منفردًا على هذا الموضوع وسمّاه بِّالمفصل في علم العربية" وهو مجموع

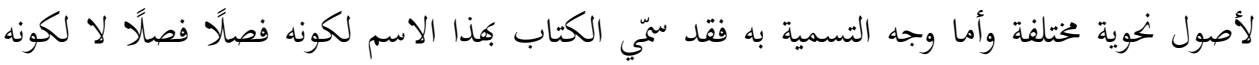
مفصّاً ضد بجمل لأنه يفضّل الإجمال. ولما أستقرّ أصولم وأتابع آرائهم أجد أن فيها نقائص عديدة. إغم راغبون في الأصول عن الأمثلة فهم

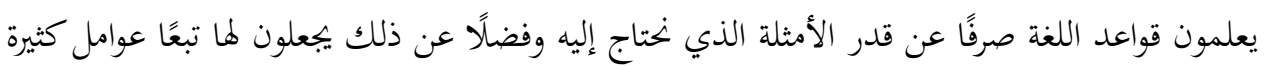

بينما كان ذلك شائعًا إذ وجدنا شخصية عملاقة تبحّرت في لغات عديدة وأخذت علومًا شتى وكانت لها اليد الطولى في النحو العربي فجدّدت هذا الفن وأضافت إلى هذا العلم إضافة مهمّة ولها آثار في ذلك وهي العلامة حميد الدين الفراهي رحه الله تعالى. آثار الإمام الفراهي في هذا الفن: - إ

لقد ترك الإمام الفراهي آثارًا عديدة في مختلف مجالات العلم والأدب والفن، أما علم النحو فقد ترك فيه

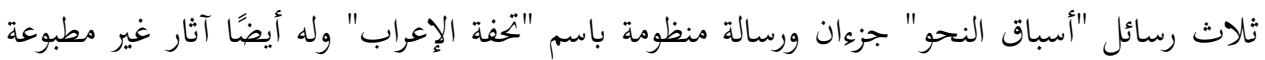

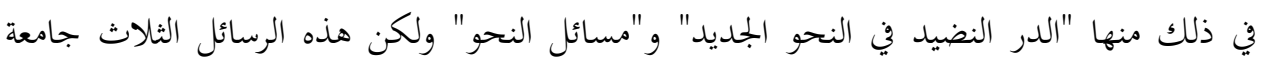

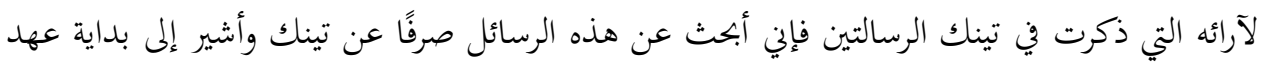
جديد في عالم النحو العربي.

\section{فيقول الإمام الفراهي وهو يذكر اختلافه:}

"قد أخذنا هذا الكتاب- أسباق النحو- من كتابي "النحو الجديد" فقد خالفنا في بعض مواضعه كتب النحو القديمة وأما سبب اختلافه بينا فهو في الكتاب المأخوذ عنه". 1 
"ولدفع الشبهة نذكر هناك اختلافين أولما أنا وضعنا حجر أساس الإعراب على تبدل أحوال الاسم

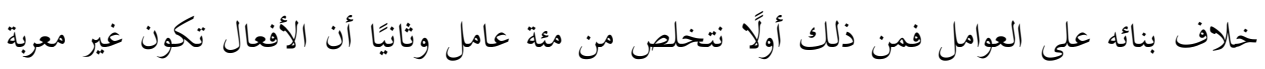

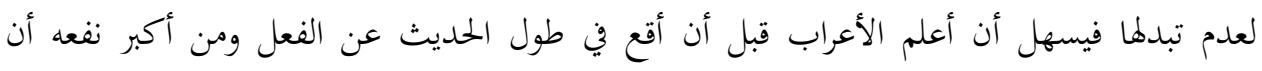

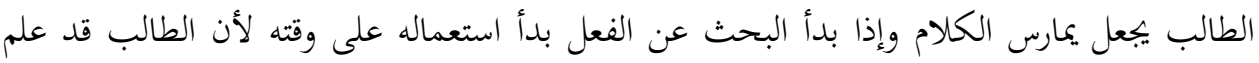

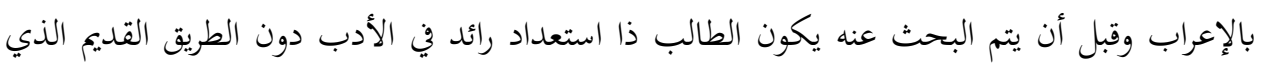

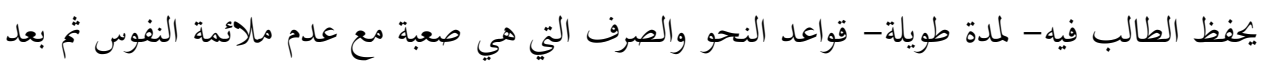

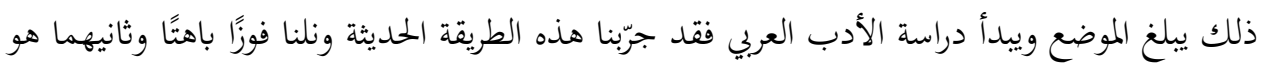

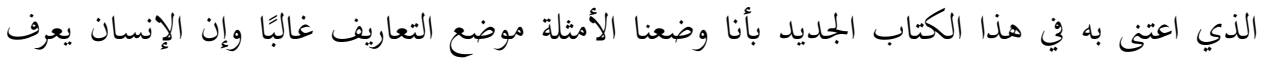
شيئًا بالتمثيل لا بالتعريف المنطقي وكثيرًا ما نرى أن المنتهي يعجز فهمه عنه فلم يوقع الطالب في صعوبة

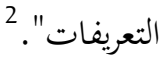

\section{وأيضًا يذكر اختلافه في الرسالة المنظومة:}

"هذه رسالة (تحفة الإعراب)، إنا وجدناها سهلًا ليحفظها الطلاب المبتدون. إن ما سلكه القدماء

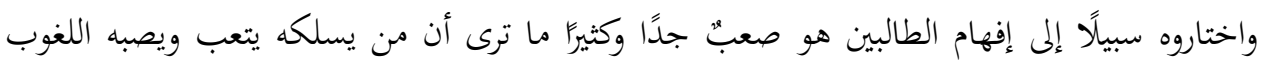

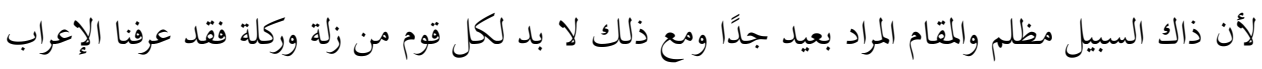
تعريفًا جديدًا ورتبنا هذا الفن ترتيبًا حديثًا فقد صارت الأفعال متخلصة من الإعراب والعوامل مجلوة عن ونس

$$
\text { البلاد فصار الفن غير صعب والطريق سهاً قصيرً". } 3
$$

\section{باب جديد في علم النحو:}

وهكذا فتح الإمام الفراهي بابًا جديدًا للتفكير في النحو العربي وهو أن لا تجعل للإعراب عوامل ولا

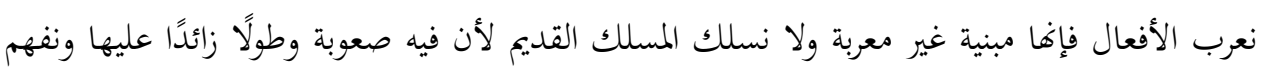
القواعد بالأمثلة ولا نميل كثيرًا إلى القواعد وتعاريفها فنكون حملاً ثقيلًا على من يطلب فيلب اللغة العربية

$$
\text { ويريد علمها وتعليمها. }
$$

ولمزيد التوضيح آي بأمثلة عديدة وأختم هذا البحث وأدعو أهل التحقيق إلى أن يحذوا حذو الإمام الفراهي ويبلغوا المرام لأنه قد خط لنا الأعلام وسهل الطريق إليه ومن جدّ وجد. 
ذكرت أن الإمام الفراهي خالف القدماء في كون العوامل سببًا للإعراب بل هو تبديل الأحوال فقط فهو يذكر أحوال الاسم وينفي العوامل عن بلادها: "إن تبدل الأحوال لهو تبدل المنازل فافهم تبدل المنازل تعلم تبدل الأحوال: إن الاسم حينما يركّب يكن له ثلاث منازل: الأولى: حينما كان جزءًا ضروريًا للكلام ك"زيدٌ ذاهبُّ"

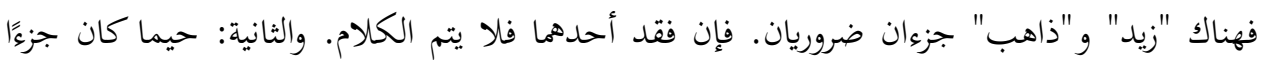

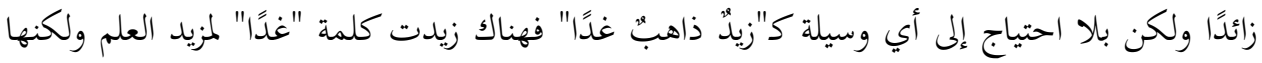

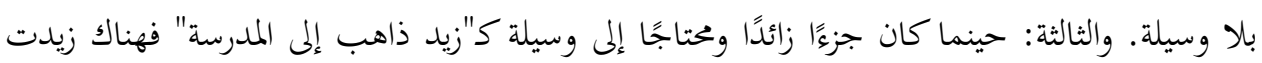
كلمة "المدرسة" لمزيد العلم ولكنها لم تلتحق بالكلام إلا بوسيلة. ويقال لهذه المنازل على الترتيب الرفع والنصب والجر والأسماء التي تحتمل هذه المنازل يقال لها المرفوع والمنصوب والمجرور. والآن نعرف هذه المرفوع والمنصوب والمجرور بأن ذاك الاسم في حالة الرفع أو النصب أو الجر أو في محل الرفع أو النصب أو الجر. وبالجملة فإن الاسم من حيث تركيبه على ثلاثة أحوال: الرفع والنصب والجر فقد وجدت أن في آخر آخر المرفوع وفي آخر المنصوب" وفي آخر المجرورٍ فكأن هذه التبدلات أعلام للأحوال وعلامات لهات لها". 4

\section{ويذكر أدوات الإشارة ولا يشير إلى عواملها فهو مخالف ويقول:}

"أدوات الإشارة هي: هذا، هذه، ذلك، تلك والمثنى هذان، هاتان، ذانك، تانك، والجمع: هؤلاء

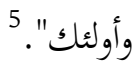
ويذكر الأمثلة ولا يقول شيئًا: "هذا القلم نفيس. وهذه الدواة رديئة. هذا الكتاب كامل. وهذه الرسالة ناقصة. ذلك الطفل شرير. وتلك البنت صالحة. هذان الطفلان أخوان. وهاتان البنتان أختان. ذانك الرجلان مسافران. وتانك المرءتان غرييتان. هؤلاء قوم زيد. وأولئك أصحاب عمرو. جواب هذين الطفلين صحيح. قول ذينك الرجلين صادق. خط هاتين البنتين جميل. عم تينك الأختين تاجر". 
قد ذهبوا إلى أن الأفعال معربة أيضًا وأما الإمام الفراهي فله أصل واحد وهو أن الأفعال لا تبدل أحوالها

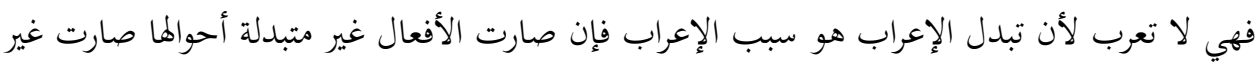
معربة فهو يقول: "إن الفعل ولو كان معروفًا أو مجهولًا لا بد من أن يكون تارة غائبًا وأخرى حاضرًا وتارة متكلمًا وأخرى

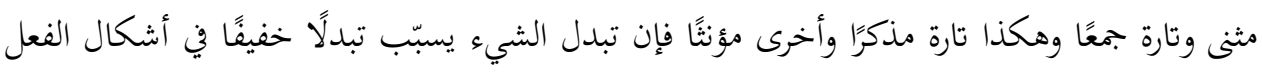

$$
\text { وهذا هو المسمّى ب"الصرف". }
$$$$
\text { ولضيق الحجم أصرف عن ذكر الأمثلة ومن يردها فليراجع نفس الكتاب. }
$$

\section{3. العناية بالأمثلة:}

قد ذكرت أنه كان في مسلك القدماء نقص هو أفم مائلون إلى التعاريف المنطقية ولا ينظرون إلى

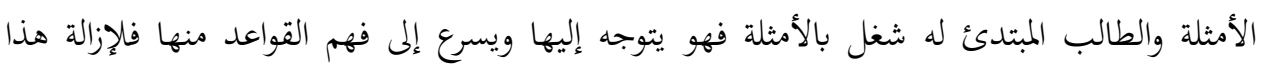

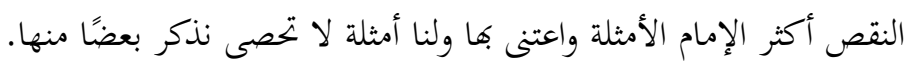
يذكر المسند إليه والمسند فهو يأتي بالأمثلة ولا يذكر التعريف: "سليم عاقل ونسيم غافل. بشير فارغ ونذير مشغول. وحيد غائب وبجيد حاضر. رشيد أمير وجعفر وزير . هاشم غني وحاتم سخي. "الإنسان حاكم. والحيوان محكوم. العالم عزيز والجاهل ذليل. المجلس قريب. والمكتب بعيد. الطعام حاضر . والخادم غائب. السؤال سهل. والجواب مشكل. الامتحان قريب. والتعطيل بعيد". 8 وكذا يذكر الحال: - 20 - n

"وحيد في البيت مريضًا. حامد أفصح القوم خطييًا. سليم كاتب جالسًا. زيد مضروب مشدودًا. ذهابي

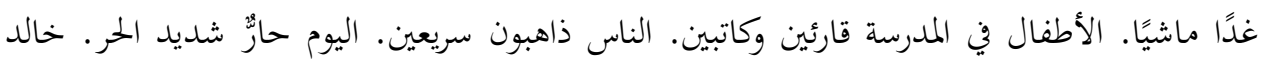
ورشيد نائمان ساكتين".

وهكذا يذكر المواقع للتعريف والتنكير: 
"هذا القلم النفيس لسعيد. لرشيد قلم نفيس. إن وحيدًا في المدرسة. في البيت خادم وأطفال. إن في

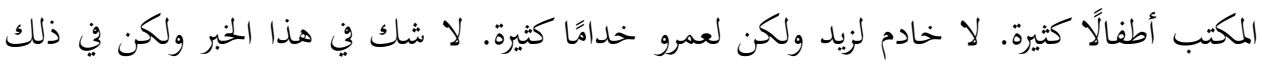

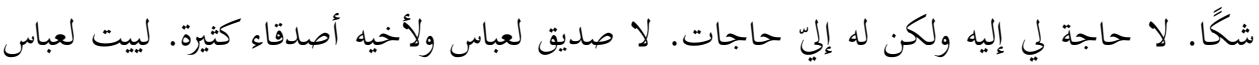
صديقًا واحدًا". ولنا أمثلة كثيرة لهذا البحث ولمن يرد مزيدًا منها فليراجع نفس الكتاب.

\section{بعض آرائه البديعة:}

وقبل أن أختم البحث لا مناص لي من أن أذكر بعض آراءه التي خالف فيها الإمام النحويين القدماء

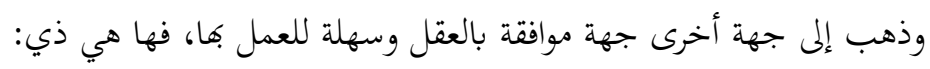

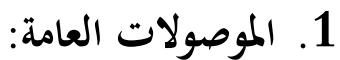

قد ذهبوا إلى أن "يوم" وأمثاله يأتي بعد الفعل مضاف إليه ففي قوله تعالى: "هذا يوم ينفع الصادقين صدقهم"، "يوم" مضاف إلى الجملة". 11 وأما الإمام الفراهي فهو ذاهب إلى أن "يوم" وأمثال ذلك لا يضاف إلى الجملة بل هو اسم للموصول وأما الذي يأتي من بعده فهو صلة له كما يقول وهو يذكر الأمثلة تحت عنوان "الموصولات العامة": "الكتاب جليسك حيث لا جليس لك، أنا مسافر غدًا إذ الناس ذاهبون للحج". 12

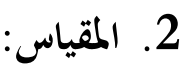

إن لكل كلمة حروفًا أصيلة يقال لها "المادة" وملا تقبل الحركة ويلتحق بها بعض الحروف الزوائد فتصير صورة جديدة فهي سمّيت بد"الوزن" كما "ح"، ب، لـ" منها حبل وبمعه حبال ووزنه عظم وبمعه عظام

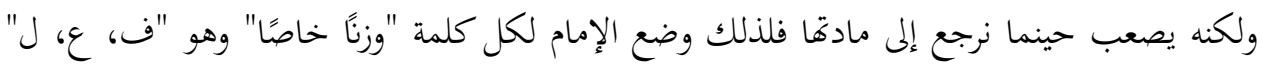

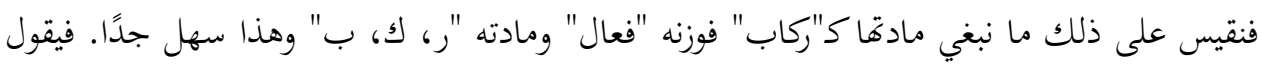
الإمام الفراهي:

"إن لكل كلمة حروفًا أصيلة يقال لها "مادة تلك الكلمة" لما تقبل هذه الكلمة الحركة أو في بعض الأحيان يلتحق بها حروف زائدة فهي تصير صورة جديدة يقال لها "وزن تلك الكلمة" ككلمة 
"الكتاب" ومادتا "ك، ت، ب" ووزها "فعال" فلبيانه خصصنا "ف، ع، ل" والأحرف الأصيلة ثلاثة غالبًا فنضع "ف، ع، ل" في موضعها ولا نتعرض الحركات والأحرف الزائدة". 13

وهكذا نقيس في علم الاشتقاق ونعلم بما في الكلمة من المادة وهذا سهل جلًا دون ما وضعوا من

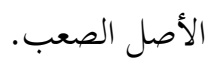

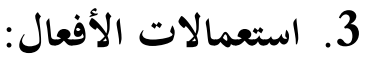

فإذا علمنا أن الفعل لا يعرف وأنه لا يؤثره أي عامل فقد زال أثر الكلمات التي ذكروها ومّموها بنواصب المضارع وجوازمه وكيت كيت. فالإمام الفراهي يخالف وذكرها تحت عنوان "استعمالات الأفعال" فيقول: "إنه تأتي قبل الفعل الماضي- هكذا قبل كل فعل كما يظهر من التفصيل الذي ذكر من بعد- أحرف لا يتبدل بها شكل الماضي وتوجد هناك تبدلات معنوية". 14

الحخاتمة:

بدا من هذا البحث الوجيز أن الإمام الفراهي كان من كبار علماء النحو الهنود وأنه طوّر هذا الفن كما

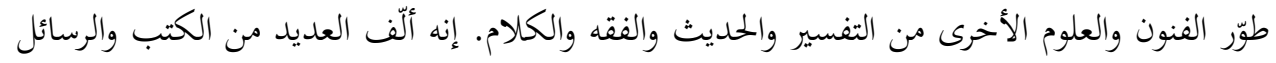

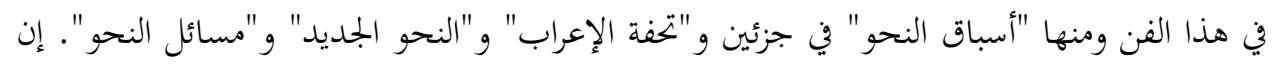

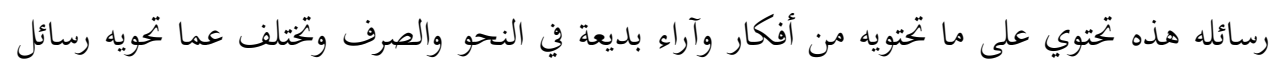

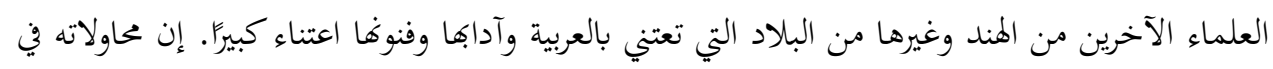

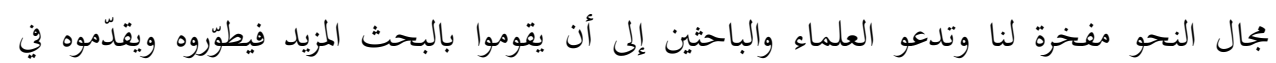
أسلوب جديد وطريقة بديعة سهلة.

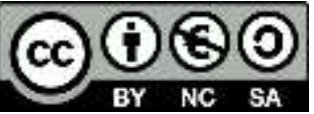

This work is licensed under a Creative Commons Attribution-NonCommercial-ShareAlike 4.0 International (CC BY-NC-SA 4.0) 
1 الأستاذ حميد الدين الفراهي: أسباق النحو، 4/1-5، مطبعة تانده، فيض آباد، د.ت

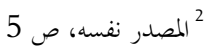

3 الإمام الفراهي: تحفة الإعراب، ص 5 5، مطبعة الدائرة الحميدية، سرائ مير، د.ت

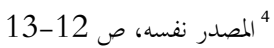

"المصدر نفسه، ص 37

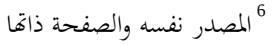

أسباق النحو، 11/2

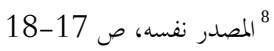

"المصدر نفسه، ص 21

10 10

11" الإمام الزخشري: المفصل في علم العربية، ص 96، دار الجيل، د.ت صل

12 13

13

14 أسباق النحو، 15/2 